\title{
Indirect Imaging of Nonradial Pulsations in a Rapidly Oscillating Ap Star ${ }^{1}$
}

\author{
Oleg Kochukhov \\ Institut für Astronomie, Universität Wien, Türkenschanzstraße 17, 1180 Wien, Austria \\ kochukhov@astro.univie.ac.at
}

\begin{abstract}
Many types of stars show periodic variations of radius and brightness, which are commonly referred to as 'stellar pulsations'. Observed pulsational characteristics are determined by fundamental stellar parameters. Consequently, investigations of stellar pulsations provide a unique opportunity to verify and refine our understanding of the evolution and internal structure of stars. However, a key boundary condition for this analysis - precise information about the geometry of pulsations in the outer stellar envelopes - has been notoriously difficult to secure. Here we demonstrate that it is possible to solve this problem by constructing an 'image' of the pulsation velocity field from time series observations of stellar spectra. This technique is applied to study the geometry of nonradial pulsations in a prototype magnetic oscillating (roAp) star HR 3831. Our velocity map directly demonstrates an alignment of pulsations with the axis of the global magnetic field and reveals a significant magnetically induced distortion of pulsations. This observation constitutes a long-sought solution of the problem of the pulsation geometry of roAp stars and enables very stringent tests of the recent theories of stellar magneto-acoustic oscillations.
\end{abstract}

Subject headings: line: profiles — stars: chemically peculiar — stars: imaging — stars: individual (HR 3831) — stars: oscillations

\section{Introduction}

A classical solution (Unno et al. 1989) of hydrodynamic equations expresses monoperiodic nonradial pulsational fluctuations of an idealized spherically symmetric star in terms

\footnotetext{
${ }^{1}$ Based on observations obtained at the European Southern Observatory, La Silla, Chile
} 
of a spherical harmonic function. Approximations of this simple stellar pulsation theory are adequate for a significant fraction of oscillating stars and, therefore, historically investigations of stellar pulsation geometry were limited to assigning spherical harmonic "quantum numbers' $\ell$ and $m$ to each observed pulsation mode. This problem is solved by several varieties of the mode identification technique (Aerts, De Pauw, \& Waelkens 1992; Watson 1988), which, with different degrees of success, are applied to photometric and spectroscopic observations of pulsating stars. However, pulsation modes in real stars are often significantly distorted by rapid stellar rotation (Lee \& Saio 1990) and global magnetic field (Dziembowski \& Goode 1985; Bigot \& Dziembowski 2002; Saio \& Gautschy 2004). These effects result in a considerably more complicated pulsation geometry, irreducible to a single spherical harmonic function. Up to now no objective technique was available for a comprehensive and reliable analysis of such distorted nonradial pulsations, but it was understood that a detailed study of high-resolution stellar spectra is the most promising approach to resolve these difficulties.

In general, all inhomogeneities in the outer layers of stellar envelopes, including pulsational disturbances, produce distortions in the stellar line profiles. As a star rotates and starspots appear and disappear from the visible hemisphere, spectral line shapes and strengths are modulated periodically. Doppler imaging (DI, e.g., Vogt, Penrod, \& Hatzes 1987 ) is a powerful technique to decipher rich information about the physics and geometry of the surface structures contained in the stellar spectrum variability. The Doppler mapping method is based on the resolution of the stellar surface provided by the effect of stellar rotation on the shapes of absorption spectral lines. If the projected rotational velocity of a star is large enough for the shapes of spectral lines to be dominated by the rotational Doppler broadening, there exists a well-defined mapping relation between distortions in a line profile and positions of the corresponding inhomogeneities on the stellar surface. Thus, a single Doppler-broadened line profile represents a one-dimensional projection of the visible stellar hemisphere, with all the structure blurred along the lines of constant Doppler shift. Spectroscopic observations at different rotation phases can be used to monitor the evolution of dips and bumps in line profiles, thus obtaining a set of non-redundant projections of a stellar surface map, and eventually to reconstruct a two-dimensional image from a time series of line profile observations. This indirect stellar surface imaging method was first applied to the problem of mapping chemical inhomogeneities in early-type stars (Khokhlova, Rice, \& Wehlau 1986) and was subsequently extended to investigation of temperature spots (Vogt et al. 1987) and complex magnetic topologies (Semel 1989) in active late-type stars and global magnetic fields (Piskunov \& Kochukhov 2002) in chemically peculiar stars. Vogt \& Penrod (1983) have emphasized that rotational broadening of stellar spectra also aids in probing the horizontal structure of nonradial pulsations. However, subsequent spectroscopic studies of rapidly rotating oscillating stars (Gies \& Kullavanijaya 1988; Kennelly, Walker, \& Mer- 
ryfield 1992; Hao 1998) used only resolution of pulsation structures along stellar longitudes and restrictively approximated velocity field with a single spherical harmonic.

In a recent development Berdyugina, Telting, \& Korhonen (2003) presented a new exploration of the capabilities of the DI technique in its application to mapping of stellar nonradial pulsations. These authors employed a temperature DI code in modeling pulsational line profile variations and assumed that pulsational fluctuations at the stellar surface can be represented by a rotation of a fixed pattern. As emphasized by Kochukhov (2004), the latter assumption restricts pulsational mapping to the reconstruction of a spherical harmonic geometry, which is aligned with the stellar rotation axis and is characterized by a unique non-zero azimuthal number $m$. This condition is not fulfilled for zonal modes and is invalid for all types of oblique stellar pulsations, hence the method of Berdyugina et al. (2003) cannot be applied to study oscillations of roAp stars.

Here we present the first application of an alternative technique. In our approach the principle of Doppler imaging is extended to the reconstruction of the time-dependent velocity field. The temporal and surface variation of the velocity vector are represented with a superposition of the two constant surface distributions:

$$
\boldsymbol{V}(t, \theta, \phi)=\boldsymbol{V}^{c}(\theta, \phi) \cos (\omega t)+\boldsymbol{V}^{s}(\theta, \phi) \sin (\omega t)
$$

where $\omega$ is the pulsation frequency and $\theta, \phi$ are usual spherical coordinates on the stellar surface. The $\boldsymbol{V}^{c}$ and $\boldsymbol{V}^{s}$ vector maps are recovered directly from the observed line profile variability without imposing any specific global constraints on the pulsation geometry. This is equivalent to mapping a two-dimensional surface distribution of the pulsation amplitude and phase for each velocity component.

The foundations of the pulsational Doppler mapping and description of its computer implementation were presented by Kochukhov (2004). We refer the reader to this paper for a detailed explanation of the technique and discussion of the numerical simulations which were used to evaluate performance and intrinsic limitations of the new surface mapping method.

\section{Pulsational Doppler mapping of HR 3831}

The pulsation Doppler inversion is applied to time-resolved observations of the Nd III $\lambda 6145 \AA$ line in the spectrum of the well-known roAp star HR 3831 (HD 83368). A total of 1860 spectra of this object were obtained over the period of 11 nights using the Coudé Echelle Spectrograph fiber-linked to the Cassegrain focus of the 3.6-m telescope at the European Southern Observatory. A preliminary discovery report of the pulsational variations in individual spectral lines in HR 3831 using these observational data was published by Kochukhov 
\& Ryabchikova (2001). Our observations evenly sample the 2.851976 rotation period of the star (Kurtz et al. 1997) and are characterized by the resolving power of $\lambda / \Delta \lambda=123,000$ and the signal-to-noise ratio of 110-160 pixel $^{-1}$. A $70^{\text {s }}$ exposure time was chosen to ensure an appropriate sampling of the 11.67 oscillation period. Fig. 1 shows an example of the rapid line profile variation of HR 3831 and presents a comparison between the observed and calculated spectra.

Reconstruction of the pulsation velocity field of HR 3831 took into account an inhomogeneous surface distribution of neodymium which significantly distorts the mean Nd III line shapes. The maps of chemical abundance and pulsational fluctuations were recovered simultaneously in a self-consistent manner. The resulting $\mathrm{Nd}$ abundance map agrees very well with the distribution derived by Kochukhov et al. (2004) using a different DI code.

Nonradial pulsations in roAp stars are characterized by very small photometric amplitudes. The respective pulsational temperature variation does not exceed $\sim 10 \mathrm{~K}$ (Medupe \& Kurtz 1998). Such temperature changes do not have a noticeable effect on the stellar line profiles and can be safely neglected in our modeling.

The formation region of the Nd III $\lambda 6145 \AA$ line is located high in the atmospheres of roAp stars (Ryabchikova et al. 2002). The horizontal motion due to the high-overtone $p$-mode pulsation is expected to be either negligible (Bigot \& Dziembowski 2002) or considerably smaller (Saio \& Gautschy 2004) than the radial motion at this atmospheric height. Trial mapping of the full vector pulsation velocity distribution demonstrated that, whereas including the horizontal pulsation motion substantially increases the number of free parameters, it does not lead to an appreciable improvement of the fit to observations. Taking this into account we limited the pulsation DI reconstruction to the vertical velocity component which is represented by the $V_{r}^{c}$ and $V_{r}^{s}$ velocity amplitudes.

The Doppler maps of the pulsation velocity field of HR 3831 revealed with our technique are illustrated in Fig. 2. The $V_{r}^{c}$ map shows a clear oblique dipolar pattern with the local pulsation amplitude reaching up to $4.0 \mathrm{~km} \mathrm{~s}^{-1}$. On the other hand, the amplitude of the stuctures in the second map, $V_{r}^{s}$, does not exceed a few hundred $\mathrm{ms}^{-1}$. Such a relation of the two maps is typical of nearly axisymmetric pulsations (see Sect 5.5 of Kochukhov 2004).

\section{Discussion}

Indirect imaging of the surface structure of nonradial oscillations has important implications for stellar astrophysics. Results of the first Doppler inversion of the pulsation velocity presented here can be regarded as an ultimate solution of the long-standing problem 
of the pulsation geometry of roAp stars. Until now the high-overtone $p$-mode oscillations in these unique objects were investigated primarily with the high-speed photometric techniques. Analyses of light variations of roAp stars (Kurtz 1990) and, in particular, HR 3831 (Kurtz et al. 1997), demonstrated that the amplitude and phase of pulsations are modulated with the stellar rotation. A coincidence of the times of maxima of pulsation amplitude and magnetic field extrema was interpreted within the oblique pulsator framework (Kurtz 1982). This phenomenological model postulated that pulsations are represented by an axisymmetric dipolar $(\ell=1)$ mode inclined with respect to the stellar rotation axis and aligned with the axis of the global magnetic field. Subsequent theoretical studies were carried out by Shibahashi \& Takata (1993), Takata \& Shibahashi (1995) and, most recently, by Saio \& Gautschy (2004) assuming a dominant role of the magnetic field. These calculations suggested that important deviations of the mode geometry from the purely dipolar topology might occur, although pulsation structure was still expected to remain almost axisymmetric and aligned with the magnetic field. A totally different picture has emerged from the theoretical investigation by Bigot \& Dziembowski (2002) who focused on the essential role of the centrifugal force resulting from the stellar rotation. According to their theory pulsations in roAp stars are described by a superposition of all $\ell=1$ harmonic components and are neither axisymmetric, nor necessarily aligned with the magnetic field. Limited information content of the photometric observations did not allow astronomers to distinguish between these competing approaches to the roAp pulsation structure.

The surface velocity mapping technique is not limited to the spherical harmonic parameterization of pulsational fluctuations and can be used to explore the physics of nonradial oscillations in rotating magnetic stars. In particular, it is able to supply a crucial observational constraint required for testing the alternative theories of roAp pulsations. The pulsation map (Fig. 2) reconstructed here for the prototype roAp star HR 3831 displays an obvious correlation with the stellar magnetic field geometry. Kochukhov et al. (2004) found that the dominant dipolar component of the global field in HR 3831 is inclined by about $90^{\circ}$ relative to the stellar rotation axis and is oriented at $\approx 180^{\circ}$ in longitude. At the same time, the $V_{r}^{c}$ velocity map presented in Fig. 2 indicates that pulsation geometry of HR 3831 is dominated by an axisymmetric structure. Furthermore, it is clear that the two maxima of the pulsation amplitude coincide with the magnetic poles. This is the first independent verification of the alignment of pulsations and magnetic field in oscillating magnetized stars.

Our pulsational mapping of HR 3831 suggests a dominant role of the magnetic perturbation of the $p$-modes and considerably less important influence of the stellar rotation. Indeed, the pulsation Doppler image of the surface velocity field reveals no significant asymmetry which can be attributed to the oblique $\ell=1,|m|=1$ components. Therefore, we conclude that pulsations in HR 3831 cannot be described by the theory brought forward by Bigot 
\& Dziembowski (2002), who attempted to fully take into account the rotationally induced mode perturbations but limited their calculations to a relatively weak magnetic field.

Fig. 3 illustrates an analysis of the dominant axisymmetric pulsation structure reconstructed with our technique. This figure shows the latitudinal dependence of the $V_{r}^{c}$ pulsation amplitude in the reference frame of the stellar magnetic field. The inferred latitudinal trend is clearly different from the one expected for a pure oblique $\ell=1$ pulsation. Axisymmetric multipolar decomposition of the observed latitudinal dependence suggests that oscillations in HR 3831 can be roughly described with a superposition of $\ell=1$ and $\ell=3$ harmonic components. The octupolar component has the same sign as the dipolar contribution and an amplitude smaller by a factor of two $\left(\mathrm{A}_{\ell=3} / \mathrm{A}_{\ell=1}=0.50 \pm 0.04\right)$. In other words, we discover that pulsations in HR 3831 are strongly confined to the magnetic field axis. This pulsation geometry is in remarkable agreement with the predictions obtained in recent calculations assuming the dominant role of the magnetic field (Saio \& Gautschy 2004). This concordance of the empirical model and theory documents a significant breakthrough in our understanding of the physics of $p$-mode oscillations in magnetic stars and possibly confirms an important role of damping by the magnetic slow waves investigated by Saio \& Gautschy (2004) in the context of excitation of the roAp-type pulsations.

In addition to revealing the true pulsation geometry of roAp stars, our precise velocity map recovered for HR 3831 opens numerous possibilities for asteroseismological determination of various stellar parameters. For instance, Takata \& Shibahashi (1995) argued that the presence of a large quadrupolar component in the magnetic field geometry is expected to induce $\ell=0$ and $\ell=2$ axisymmetric structures in the pulsation velocity distribution. However, Fig. 3 convincingly demonstrates that the even multipolar components are very weak in HR 3831. Hence, the magnetic field of this star must be basically dipolar - a conclusion obtained independently from an analysis of any classical magnetic field observables.

This work was supported by the Lise Meitner fellowship granted by the Austrian Science Fund (FWF, project No. M757-N02). We acknowledge extensive use of the SIMBAD database, operated at CDS, Strasbourg, France.

\section{REFERENCES}

Aerts, C., De Pauw, M., \& Waelkens, C. 1992, A\&A, 266, 294

Berdyugina, S. V., Telting, J. H., \& Korhonen, H. 2003, A\&A, 406, 273

Bigot, L., \& Dziembowski, W. A. 2002, A\&A, 391, 235 
Dziembowski, W., \& Goode, P. R. 1985, ApJ, 296, L27

Gies, D. R., \& Kullavanijaya, A. 1988, ApJ, 326, 813

Hao, J. 1998, ApJ, 500, 440

Kennelly, E. J., Walker, G. A. H., \& Merryfield, W. J. 1992, ApJ, 400, L71

Khokhlova, V. L., Rice, J. B., \& Wehlau, W. H. 1986, ApJ, 307, 768

Kochukhov, O. 2004, A\&A, 423, 613

Kochukhov, O., Drake, N. A., Piskunov, N., \& de la Reza, M. 2004, A\&A, 424, 935

Kochukhov, O. \& Ryabchikova, T. 2001, A\&A, 377, L22

Kurtz, D. W. 1982, MNRAS, 200, 807

Kurtz, D. W. 1990, ARA\&A, 28, 607

Kurtz, D. W., van Wyk, F., Roberts, G., Marang, F., Handler, G., Medupe, R., \& Kilkenny, D. 1997, MNRAS, 287, 69

Lee, U., \& Saio, H. 1990, ApJ, 349, 570

Medupe, R., \& Kurtz, D. W. 1998, MNRAS, 229, 371

Piskunov, N., \& Kochukhov, O. 2002, A\&A, 381, 736

Ryabchikova, T. A., Piskunov, N. E., Kochukhov, O., Tsymbal, V., Mittermayer, P., \& Weiss, W. W. 2002, A\&A, 384, 545

Saio, H., \& Gautschy, A. 2004, MNRAS, 350, 485

Shibahashi, H., \& Aerts, C. 2000, ApJ, 531, L143

Shibahashi, H., \& Takata, M. 1993, PASJ, 45, 617

Semel, M. 1989, A\&A, 225, 456

Takata, M., \& Shibahashi, H. 1995, PASJ, 41, 219

Unno, W., Osaki, Y., Ando, H., Saio, H., \& Shibahashi, H. 1989, Nonradial Oscillations of Stars, (2d ed., Tokyo: University of Tokyo Press)

Vogt, S. S., \& Penrod, G. D. 1983, ApJ, 275, 661 
Vogt, S. S., Penrod, G. D., \& Hatzes, A. P. 1987, ApJ, 321, 496

Watson, R. D. 1988, Ap\&SS, 140, 255 

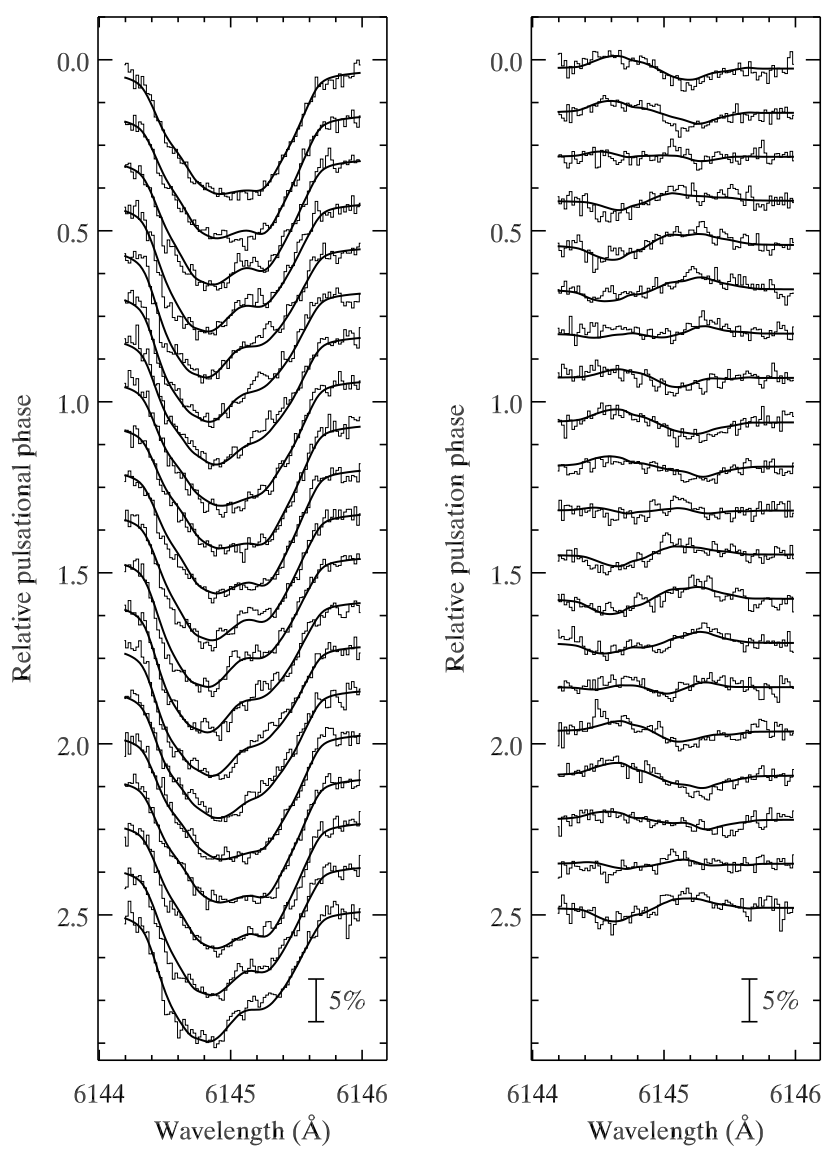

Fig. 1.- Observed variation of the Nd III $\lambda 6145 \AA$ line (histogram) is compared with the best fit synthetic spectra (solid line). The left panel shows the observed and synthetic spectra for 20 phases (out of 1860 used in the inversion) close to the maximum of pulsational fluctuations (rotation phases $\varphi=0.966-0.973$ according to the ephemeris of Kurtz et al. 1997). The right panel presents the difference between individual profiles and the average line shape. The spectra for consecutive pulsation phases are shifted in the vertical direction. The bars at the bottom of the panels show the vertical scale in units of the continuum flux. 

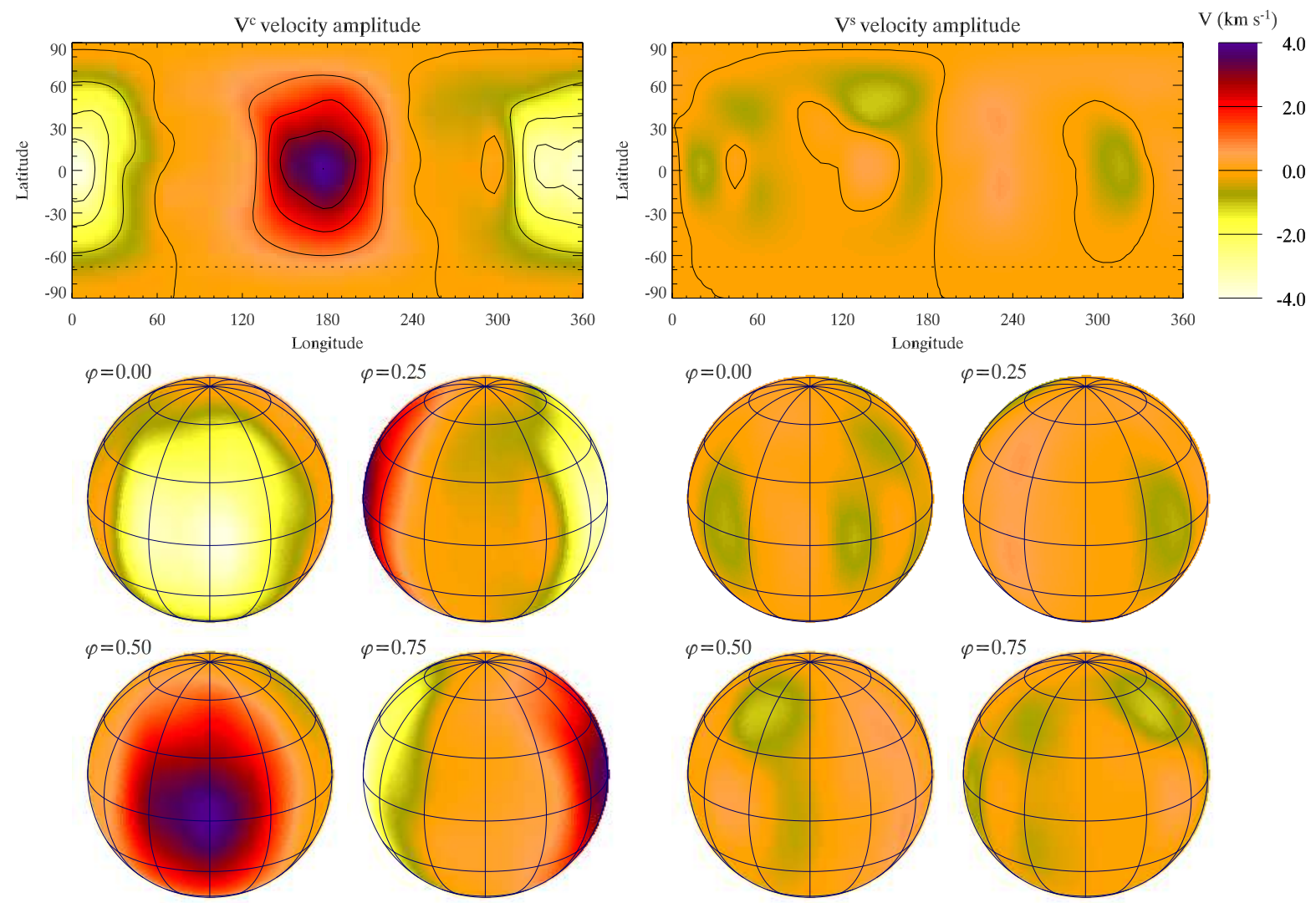

Fig. 2.- Results of the first Doppler imaging reconstruction of the stellar pulsation velocity field. Rectangular panels show the $V_{r}^{c}$ (left) and $V_{r}^{s}$ (right) vertical velocity amplitude maps. The contours of equal velocity are plotted over the greyscale images with a step of 1.0 $\mathrm{kms}^{-1}$. The horizontal dotted line shows the lowest visible latitude corresponding to the angle between the stellar axis of rotation and the observer's line of sight ('inclination angle', $i=68^{\circ}$ ) adopted in the analysis of HR 3831. The plots below present spherical projection of the respective pulsation velocity maps. The star is shown at 4 different aspect angles corresponding to the rotation phases $\varphi=0.00,0.25,0.50$, and 0.75 . The grid at the stellar surface is plotted with a $30^{\circ}$ step in longitude and latitude. 


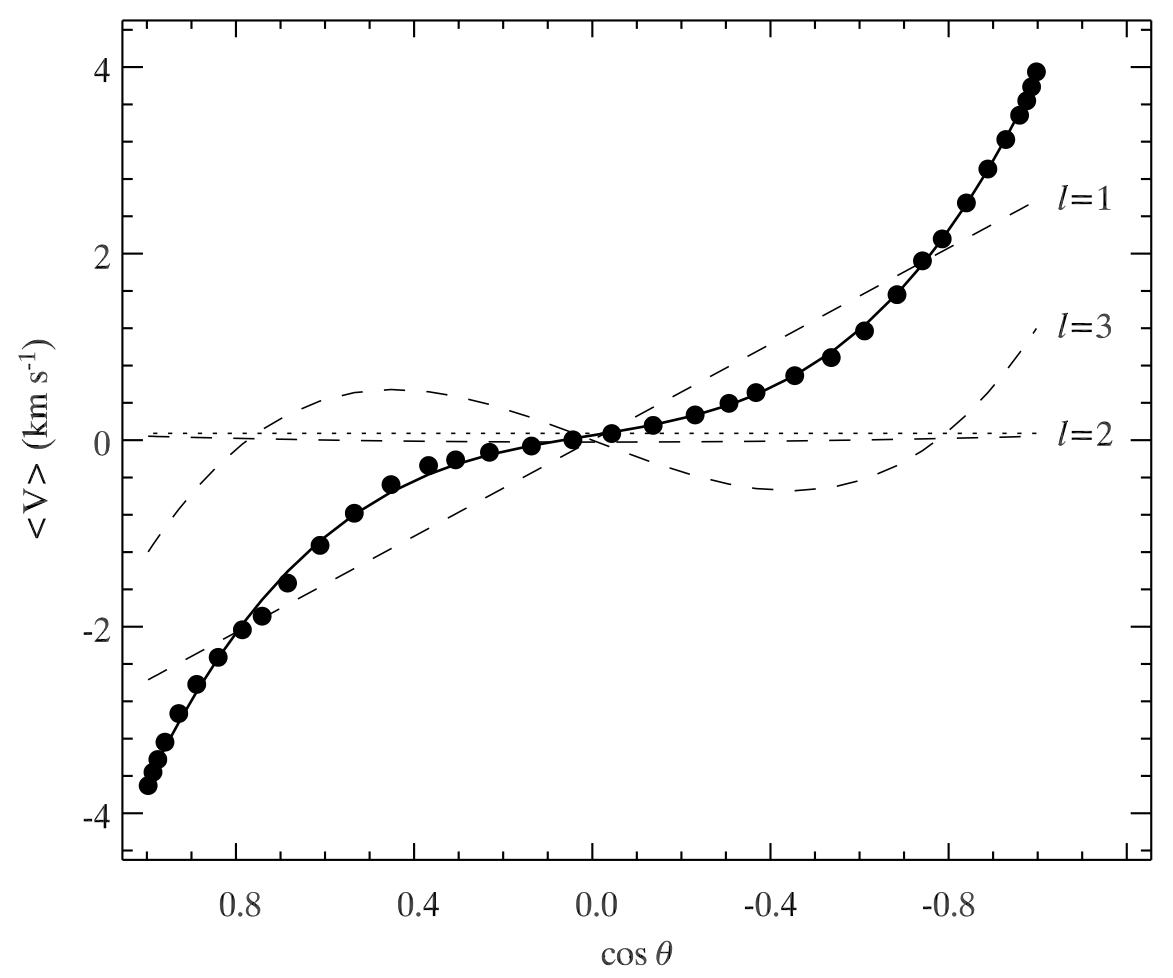

Fig. 3.- Analysis of the latitudinal dependence of the $V_{r}^{c}$ pulsation velocity amplitude. The symbols show average velocity amplitude as a function of the cosine of the angle $\theta$ counted from one of the pulsation poles. The solid line illustrates the fit with a superposition of axisymmetric $\ell=0,1,2$, and 3 spherical harmonic components. Contributions of individual multipolar components are shown by the dashed lines for $\ell=1-3$ and by the dotted line for $\ell=0$. 